\title{
On the relation between rotation, convection and activity in intermediate-mass giants
}

\author{
P. Gondoin
}

European Space Agency, ESTEC - Postbus 299, 2200 AG Noordwijk, The Netherlands

\begin{abstract}
I found evidence that the X-ray surface flux of intermediate-mass $\mathrm{G}$ and $\mathrm{K}$ giants is correlated with their rotation period and Rossby number. Confidence in the degree of correlation is significantly higher when stellar gravity is taken into account. An empirical relation is found that accounts for the X-ray luminosity evolution of single intermediate-mass giants and giants in close or long-period binary systems, such as RS CVn-type systems, as they evolve off the main sequence towards the top of red giant branch.
\end{abstract}

Keywords. Stellar activity, stellar coronae, stellar evolution, late-type stars, X-ray

\section{Introduction}

One major topic of stellar activity is to explain how phenomena seen on the Sun and stars, and especially magnetic phenomena, depend on stellar parameters such as rotation, and convection. One magnetic field diagnostic for cool stars is coronal X-ray emission. A relation between X-ray luminosities and rotation has been reported for late-type dwarfs but the connection between rotation, convection and magnetic activity is less evident among giants.

\section{Rotation and internal structure evolution of giants}

Giants with $1.5 \mathrm{M}_{\odot}<M<3.8 \mathrm{M}_{\odot}$ have early-F, A, and late B-type progenitors on the main sequence that have no deep outer convection zones and that are typically rapid rotators (Abt \& Morell 1995; Royer et al. 2002). As they evolve off the main sequence, in the shell hydrogen burning stage, they develop thin outer convection zones. These giants then rapidly traverse the $\mathrm{F}$ and $\mathrm{G}$ spectral types zone of the H-R diagram which is relatively devoid of stars and is known as "the Hertzsprung gap". During this rapid evolution, the internal structure of these stars changes substantially. The thin convective shells prior to early $\mathrm{G}$ give way to rapidly deepening convection zones at midlate $\mathrm{G}$. The increasing convection zone depth combined with fast rotation triggers dynamo processes that generate the magnetic fields that, by analogy with the Sun, cause the Xray emission of the outer stellar atmospheres. A large fraction of the yellow giants are able to hold their high rotation until mid-G spectral types. Beyond this point, rotation velocity measurements (Gray 1989) indicate a strong rotational braking among single giants. Within the frame of the solar paradigm, a correlated decrease in X-ray emission is expected.

\section{Correlation between rotation, convection, and X-ray emission}

A sample of intermediate-mass $\mathrm{G}$ and $\mathrm{K}$ giants with masses included between 1.5 and 3.8 solar mass (see Fig. 1 left) and with known rotational periods was defined from a sample of single $\mathrm{G}$ giants with known rotation periods (Gondoin 2005), from a list of 

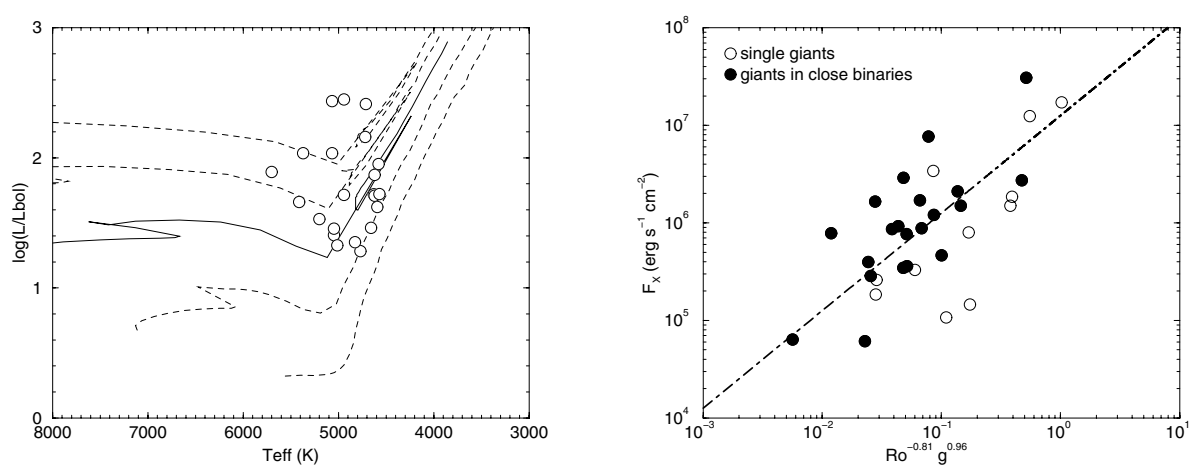

Figure 1. Left: H-R diagram of a sample of giants in close binaries compared with evolutionary tracks (Schaller et al. 1992). The lines from bottom to top describe the evolutionary tracks of $1 \mathrm{M}_{\odot}, 1.5 \mathrm{M}_{\odot}, 2 \mathrm{M}_{\odot}$ (solid line), $2.5 \mathrm{M}_{\odot}$ and $3 \mathrm{M}_{\odot}$ stars, respectively. Right: X-ray surface flux of single giants (open circle) and giants in close binary systems (filled circles) as a function of a parameter defined as $R o^{-0.81} \times\left(\mathrm{g} / \mathrm{g}_{\odot}\right)^{0.96}$, where $R o$ is the Rossby number and $\mathrm{g} / \mathrm{g} \odot$ the stellar gravity in solar units. The dot-dashed line is the best linear fit (see (3.1)).

selected binaries established by Schrijver \& Swaan (1991), from a catalog of active binary stars (Strassmeier et al. 1993), and from a list of single-lined spectroscopic binaries with orbital period shorter than 250 days (De Medeiros et al. 2002). I found evidence that the X-ray surface flux $F_{\mathrm{X}}$ of intermediate-mass $\mathrm{G}$ and $\mathrm{K}$ giants is correlated with their Rossby number $R_{\mathrm{o}}$ expressed as the ratio between the rotation period and the convective turnover time. Confidence in the degree of correlation is significantly higher when stellar gravity $g$ is taken into account. The empirical relation (see Fig. 1 right) is given by:

$$
\log \left(F_{\mathrm{X}}\right)=-0.81 \times \log \left(R_{\mathrm{o}}\right)+0.96 \times \log \left(g / g_{\odot}\right)+7.1
$$

where $F_{\mathrm{X}}$ is expressed in erg s $\mathrm{sm}^{-1}$.

\section{The effect of convection on the X-ray activity evolution of giants}

In order to calculate the X-ray luminosity evolution of single giants and of giants in long-period binary systems, I used empirical models of rotation evolution (see Fig. 2 left) of single intermediate-mass stars (Gondoin 2005). For giants in close binary systems, I assumed that the rotation period remains constant during the stellar evolution off the main the sequence to the top of the red giant branch. These rotation evolution models were combined with convection turnover times derived by Gunn et al. (1998) to estimate the evolution of the Rossby numbers of intermediate-mass stars during their evolution off the main sequence. The X-ray fluxes of these giants were then calculated from (3.1). Evolutionary models computed by Schaller et al. (1992) were used to determine the stellar radii and bolometric luminosities of the stars and to infer their X-ray luminosities and $\mathrm{X}$-ray to bolometric luminosity ratios as a function of effective temperature. The results indicate that the X-ray luminosity of single giants, including FK Comae-type stars and giants in long-period binary systems increases by three to four orders of magnitudes between the mid-F and mid-G spectral types due to a deepening of their convection envelope and then decreases sharply as the stars ascend the red giant branch due to a strong rotational braking. The results also indicate that the X-ray luminosity evolution of intermediate-mass giants in close binary systems, such as RS CVn systems, increases as they cross the Hertzsprung gap up to G spectral type, and remains approximately 

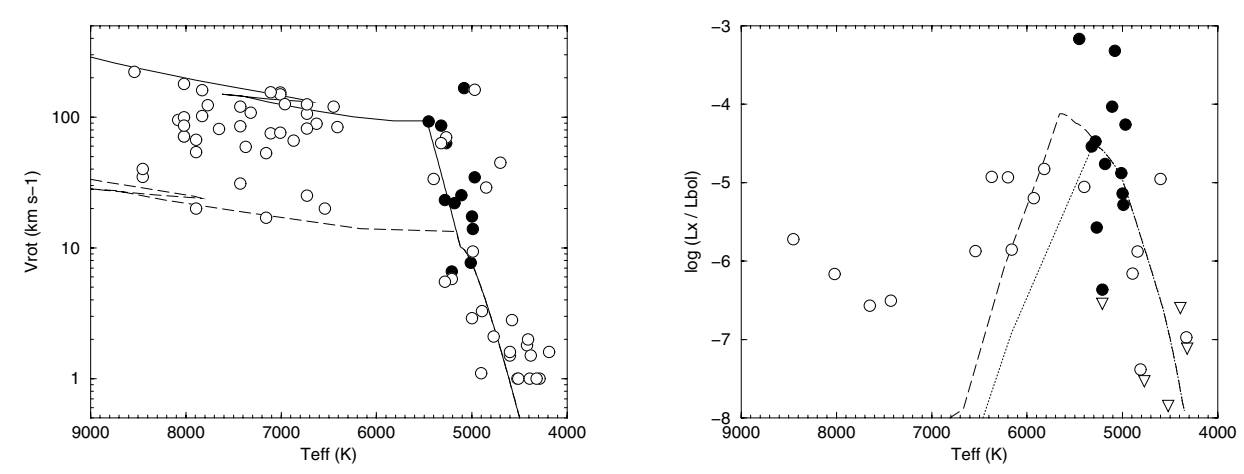

Figure 2. Left: equatorial velocity of a sample of single G giants (black circles) as a function of effective temperature. The open circles are the projected rotational velocities of A, F, G, and K single field giants. The solid lines describe rotation evolution models for $2.5 \mathrm{M}_{\odot}$ (lower curve) and $2.0 \mathrm{M}_{\odot}$ stars (upper curve) with equatorial velocities of 50 and $300 \mathrm{~km} \mathrm{~s}^{-1}$, respectively, on the main sequence. Right: X-ray to bolometric luminosity ratio as a function of effective temperature. The lines represent empirical models of $L_{\mathrm{X}} / L_{\mathrm{bol}}$ evolution for $2.5 \mathrm{M}_{\odot}$ (dotted line) and $2.0 \mathrm{M}_{\odot}$ stars (dashed line) using the rotation evolution models previously described. The triangles represent giants for which only upper limits of the X-ray luminosity are available.

constant during their further evolution. These X-ray luminosity evolution trends are supported by experimental data (see Fig. 2 right). I conclude that they most likely result from the combined effect of convection and rotation on magnetic activity.

\section{Conclusions}

- A relation exists between the rotation rate, Rossby number, and X-ray activity level in intermediate-mass giants;

- this relation accounts for the increase of the X-ray luminosity of single giants, including FK Comae-type stars and giants in long-period binary systems between the mid-F and mid-G spectral types due to a deepening of their convection envelope and for a subsequent decrease of their X-ray luminosity due to a strong rotational braking;

- the major role played by binarity in the magnetic activity level of intermediate-mass giants is to maintain rapid rotation at late stages of stellar evolution;

- gravity and mass are important stellar parameters in determining the X-ray surface flux, respectively X-ray luminosity, of late-type giants.

\section{References}

Abt, H.A. \& Morell, N.I. 1995, ApJS 99, 135

De Medeiros, J.R., Da Silva, J.R.P. \& Maia, M.R.G. 2002, ApJ 578, 943

Gondoin, P. 2005, A\& A 444, 531

Gray D.F. 1989, ApJ 347, 1021

Gunn, A.G., Mitrou, C.K. \& Doyle, J.G. 1998, MNRAS 296, 150

Hearn, A.G. 1977, Solar Phys. 51, 159

Royer, F., Grenier, S., Baylac, M.O., et al. 2002, A\&A 393, 897

Schaller G., Schaerer D., Meynet G. \& Maeder A., 1992, A\&AS 96, 269

Schrijver, C.J. \& Zwaan, C. 1991, A\&A 251, 183

Strassmeier, K.G., Hall, D.S., Fekel, F.C., et al. 1993, A\&AS 100, 173 